\title{
Universal Vaccination Guidelines and status during the Corona Era
}

\author{
Gedam D. ${ }^{1 *}$, Verma M. ${ }^{2}$ \\ DOI: https://doi.org/10.17511/ijphr.2020.i03.01 \\ 1* D. Sharad Gedam, Associate Professor, Department of Pediatrics, Government Autonomous Medical College (GMC), Associated hospital, \\ Vidisha, Madhya Pradesh, India. \\ 2 Mamta Verma, Associate Professor, College of Nursing, All India Institute of Medical Sciences, Bhopal, Madhya Pradesh, India.
}

As COVID-19 continues to spread around the world, people in all countries are being encouraged to take preventive measuress to prevent transmission, including in many countries by staying at home and physical distancing. But a pandemic does not erase other diseases and their impact. While many services, including some health services, are being scaled back, the risk of further outbreaks of infectious diseases grows. The healthcare disruptions caused by COVID-19 could have a devastating impact on child mortality.

Keywords: Covid-19, Corona Virus, Vaccine, Universal Immunization Guidelines

Corresponding Author

D. Sharad Gedam, Associate Professor, Department

of Pediatrics, Government Autonomous Medical

College (GMC), Associated hospital, Vidisha, Madhya

Pradesh, India.

Email: sharad.gedam@gmail.com
How to Cite this Article

To Browse

Gedam DS, Verma M. Universal Vaccination

Guidelines and status during the Corona Era. Public Health Rev Int J Public Health Res. 2020;7(3):26-27. Available From

https://publichealth.medresearch.in/index.php/ijphr/ article/view/139

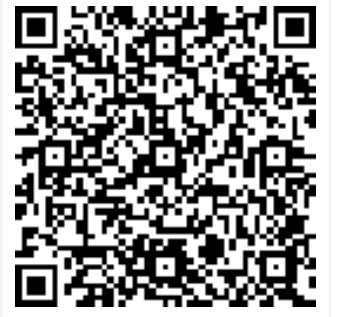

In 2020, it is estimated that 80 million children under one year of age, live in a country that has reported some kind of disturbance to the immunization program largely due to limited access to health centers, low availability of PPE for healthcare workers, and fear of contracting COVID-19. WHO is helping countries as they balance the threat of COVID-19 with the threat of vaccinepreventable disease outbreaks and deaths that could result. With global and regional guidance, as well as by enabling the delivery of essential health supplies, WHO is providing knowledge and support to immunization programs worldwide, through a precipitously evolving condition. Health officials, throughout the world, are working round the clock to save lives, in the middle of this, there are several
Important questions that need to be answered at a priority basis, what are ways to protect the most vulnerable?, what are the tools that will slow the speed of this viral transmission, and what are most feasible ways to reduce the mortality and morbidity due to Covid-19.

The number of rising questions has made the scientific and public health community recall the importance of a vaccine. While the world awaits a vaccine for Covid-19, there are a number of conditions such as measles, diphtheria, tetanus, polio among children, and pneumococcal disease and influenza among adults saves an approximate of 2-3 million lives. In India, over 200,000 children under five continue to die due to pneumonia and diarrhea, even though vaccines like the rotavirus vaccine and pneumococcal vaccine

Review Round 2

Review Round 3

Accepted

Ethical Approval

Yes

Plagiarism X-checker Note $7 \%$

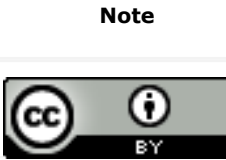
Open Access article licensed under a Creative Commons Attribution 4.0 International License https://creativecommons.org/licenses/by/4.0/ unported [CC BY 4.0]. 
Can prevent severe forms of these diseases. Immunizations are an essential health service that protects susceptible individuals from vaccinepreventable diseases (VPD) $[1,2,3]$. By providing timely immunizations, individuals and communities remain protected and the likelihood of a VPD outbreak decreases, Preventing a VPD outbreak not only saves lives but requires fewer resources than responding to the outbreak and helps reduce the burden on a health system already strained by the COVID-19 pandemic, While committing to sustaining immunization systems, countries should use approaches that respect the principle of do-noharm and limit transmission of COVID-19 while providing immunization activities, Immunization visits can also be used as opportunities to disseminate messages to encourage behaviors to reduce transmission risk of the COVID-19 virus, to identify signs and symptoms of COVID-19 disease, and to provide guidance on what to do if symptoms emerge [4]. Vaccines are particularly valuable given the disturbing rise in anti-microbial resistance (AMR) across the world. AMR occurs naturally due to environmental factors, but the surge in misuse of antibiotics-things like using antibiotics for viruses or other diseases they are not effective against, or not completing a full regimen as prescribed by a healthcare provider-has led to the emergence of "superbugs" that cannot be treated by the antibiotics we have available today. These resistant pathogens are complicating the fight against diseases like pneumococcal pneumonia, tuberculosis, and malaria. India is often referred to as the AMR capital of the world, and researchers have even called AMR "the next big pandemic." How can vaccines help limit AMR? By preventing disease and reducing the need for treatment, vaccines help lower the risk of antibiotic misuse and the development of resistance. Ensuring high immunization coverage is vital. In fact, the World Health Organization (WHO) estimates that if every child in the world received the pneumococcal conjugate vaccine or PCV-a vaccine that helps prevent a leading cause of pneumonia, meningitis, and other diseases-we could prevent an estimated 11 million days of antibiotic use each year. PCV was recently added to India's Universal Immunization Programme and is so far available in six states. Scaling up the vaccine throughout the country can go a long way in combating pneumonia and related illnesses.

Vaccines also help in developing herd
immunity

A well-known fact about vaccines is that they also lead to herd immunity in a community. We have seen this effect with the implementation of polio, Hib, measles, and pneumococcal vaccines, provided there is high coverage of the vaccine. That is, if about $80-90 \%$ of the population in a community is immunized against a disease, chances are that the remaining $10-20 \%$ unimmunized population will remain protected against the disease, as they will be surrounded by the immunized population making it unlikely for the causative organism is to get access to them. To minimize the risk of COVID-19 transmission, individuals with suspected or confirmed COVID-19 should be isolated and cared for according to WHO guidance. If a person with confirmed or suspected COVID-19 is not in a health care facility (e.g. at home), the act of seeking immunization may increase spreading infection to others, For that reason, this individual should defer vaccination until symptoms resolve, preferably following two consecutive tests negative for COVID19 (conducted 24 hours apart). If testing is not feasible, the WHO recommends deferring vaccination for 14 days after symptom resolution. If a person with confirmed or suspected COVID-19 is under care in a health care facility (e.g. inpatient) this individual should be vaccinated according to the national immunization schedule upon recovery and prior to discharge, assuming appropriate infection prevention and control measures are respected. The duration of viral shedding and transmissibility of COVID-19 is not yet well understood, As these studies become available, this guidance will be updated

\section{Reference}

01. Guiding principles for immunization activities during the COVID-19 pandemic. Available at [Article][Crossref]

02. COVID-19- Strategic Planning and Operational Guidance for Maintaining Essential Health Services During an Outbreak, 20 March 2020. Available at [Article][Crossref]

03. Critical preparedness, readiness, and response actions for COVID-19. Available at [Article][Crossref]

04. Revisiting the value of vaccination in face of COVID-19. Available at [Article][Crossref] 